\title{
High Contrast and Fast Response Polarization- Independent Reflective Display Using a Dye-Doped Dual-Frequency Liquid Crystal Gel
}

\author{
Yi-Hsin Lin \\ Hongwen Ren \\ Sebastian Gauza \\ Yung-Hsun Wu \\ Ying Zhou \\ Shin-Tson Wu \\ College of Optics and Photonics, University of Central Florida, \\ Orlando, Florida, USA
}

We have developed a new guest-host reflective display using a dye-doped dualfrequency liquid crystal (DFLC) gel. By combining dye absorption and maximal light scattering, the display is polarization independent and exhibits a high contrast ( 230:1). The high reflectivity $(R \sim 50 \%)$ is because of the vertical aligned structure at $0 V_{r m s}$. The fast response time $(\sim 5 \mathrm{~ms})$ is also fast due to the frequency modulation. A black and white segmented-alphabet reflective display prototype is also demonstrated.

Keywords: dual frequency liquid crystal; gel; guest-host reflective display; high contrast; polarization-independent

\section{INTRODUCTION}

Guest-host liquid crystal displays (GH LCDs) do not require any polarizer; therefore, they exhibit a high brightness and wide viewing angle $[1,2]$. Several device configurations such as Cole-Kashnow cell [3], White-Taylor cell [4], or double orthogonal cells [5-7] have been proposed for achieving polarization independence. A typical reflectance of the GH LCD is $\sim 50 \%$, but the contrast ratio is only $\sim 5: 1$, limited by the dichroic ratio (typically $\sim 10: 1$ ) of the employed dyes. Increasing

This work was supported by Toppoly Optoelectronics (Taiwan).

Address correspondence to Yi-Hsin Lin, College of Optics and Photonics, University of Central Florida, Orlando, Florida 32816, USA. E-mail: swu@mail.ucf.edu 
the LC cell gap or dye concentration can improve the contrast ratio; however, the reflectance is sacrificed accordingly.

Scattering is another polarization independent light modulation mechanism. A well-known example is polymer-dispersed liquid crystal (PDLC) $[8,9]$. However, the light scattering state is translucent, not black. For a high contrast display, a good black state is required. To achieve a black state, a few percent of black dyes are added and a $\sim 10: 1$ contrast ratio is demonstrated using a PDLC in a twisted cell [10]. The response time of such a guest-host PDLC is $\sim 20-30 \mathrm{~ms}$, depending on the cell gap and the LC and dye materials employed.

To further improve response time and light scattering efficiency, our group has developed a polarization independent dual-frequency liquid crystal (DFLC) gel [11]. The gel is also a light scattering device. Fast response time and high contrast ratio are the two important features of the DFLC gel. This gel has been used for high speed photonic devices, but for display applications we need to obtain a good black state, high contrast ratio, and wide viewing angle.

In this paper, we demonstrate a new GH LCD using a dye-doped DFLC gel to realize polarizer-free, fast-response, and high-contrast reflective displays. This is a normally white display utilizing the multiple light scattering from the gel together with the absorption from the dye molecules. In the voltage-off state, the display exhibits a $\sim 50 \%$ reflectance while at $\sim 30 \mathrm{~V}_{\text {rms }}$ a good black state is observed. The device contrast ratio as high as $\sim 230: 1$ is obtained and the response time is $\sim 6 \mathrm{~ms}$.

\section{SAMPLE PREPARATION, MECHANISM AND EXPERIMENTAL SETUP}

A DFLC mixture we used consists of some biphenyl esters and lateral difluoro tolanes. The formulated DFLC mixture has following physical properties: birefringence $\Delta \mathrm{n}=0.267$ (at $\lambda=633 \mathrm{~nm}, \mathrm{~T}=21^{\circ} \mathrm{C}$ ), crossover frequency $f_{c}=10 \mathrm{kHz}$, and dielectric anisotropy $\Delta \varepsilon=7.72$ at $f=1 \mathrm{kHz}$ and $\Delta \varepsilon=-3.51$ at $f=50 \mathrm{kHz}$. We mixed the DFLC, a diacrylate monomer (bisphenol-A-dimethacrylate), and the dichroic dye S428 (Mitsui Chemicals Inc.) at 90:5:5 $\mathrm{wt} \%$ ratios. The mixture was then injected into an empty cell whose inner surfaces were coated with a thin indium-tin-oxide (ITO) electrode. The cell gap is $d=5 \mu \mathrm{m}$. The filled cell was irradiated by a UV light $\left(\lambda \sim 365 \mathrm{~nm}, \mathrm{I} \sim 15 \mathrm{~mW} / \mathrm{cm}^{2}\right)$ at room temperature for $1 \mathrm{~h}$ with $\mathrm{a} \sim 40 \mathrm{~V}_{\text {rms }}$ biased voltage $(f=$ $1 \mathrm{kHz})$. The formed chain-like polymer networks are along the electric field direction because the LC directors are aligned 


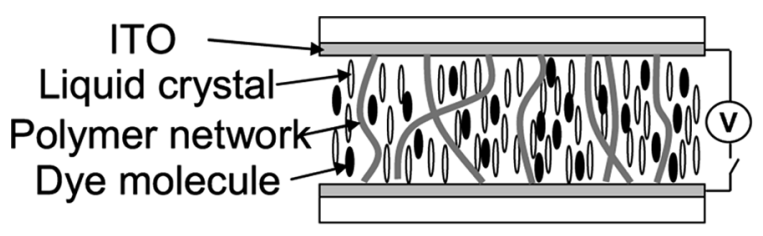

(a)

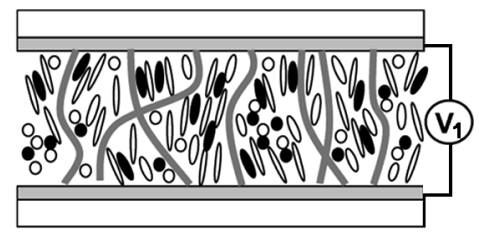

(b)

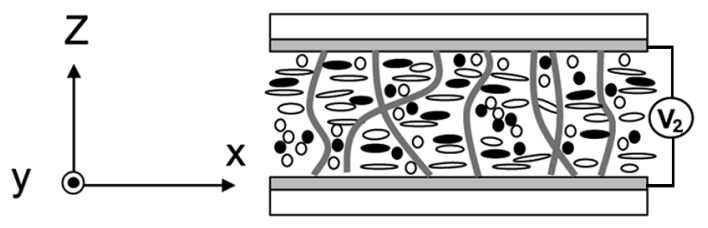

(c)

FIGURE 1 Schematic representation of the operating principle. (a) Voltage-off state, (b) voltage-on state at $f>f_{\mathrm{c}}$, and (c) voltage-on state at $f>f_{\mathrm{c}}$ and $\mathrm{V}_{2}>\mathrm{V}_{1}$.

perpendicular to the glass substrates during the UV curing process, as shown in Figure 1(a).

The light modulation mechanism of the dye-doped DFLC gel can be schematically depicted in Figures 1(a), (b) and (c). At V $=0$, the cell does not scatter light and the absorption is rather weak because the dye molecules are aligned perpendicular to the substrates, as shown in Figure 1(a). Therefore, the display has the highest reflectance. When the applied high-frequency $\left(f>f_{c}\right)$ voltage exceeds a threshold, the LC directors and dye molecules are tilted away from the electric field because the DFLC has a negative $\Delta \varepsilon$. Two kinds of light loss mechanisms take place: 1 ) light scattering due to the LC domain reorientations and refractive index mismatch, and 2) absorption due to the black dyes. In a high voltage state, the dye molecules are reoriented perpendicular to the electric field direction so that they absorb the incoming light leading to a black state, as Figure 1(b) shows. Since 
the ITO substrates have no alignment treatment, the absorption has no preferred direction. As the applied voltage increases further, the liquid crystals and dye molecules are reoriented in the $x-y$ plane, as Figure 1(c) depicts, so that the gel's light scattering and the dye's absorption reach their maxima and the display appears black.

To measure the reflectance of the dye-doped DFLC gel, a linearly polarized green diode laser $(\lambda=532 \mathrm{~nm})$ was used for characterizing the device performances. A dielectric mirror was placed behind the cell so that the laser beam traversed through the cell twice. A computer controlled LabVIEW data acquisition system was used for driving the sample and recording the light reflectance.

\section{EXPERIMENTAL RESULTS AND DISCUSSION}

The voltage-dependant reflectance of the dye-doped DFLC gel is plotted in Figure 2. The reflectance is normalized to that of a pure DFLC cell with the same cell gap. At $f=1 \mathrm{kHz}$, the reflectance does not change with the applied voltage. That is because the applied voltage does not reorient the dye-doped DFLC gel at $f=1 \mathrm{kHz}$ when the LC directors with a positive $\Delta \varepsilon$ are in the homeotropic structure. At $f=50 \mathrm{kHz}$, the LC directors with negative $\Delta \varepsilon$ are reoriented by the applied electric field; therefore, the reflectance remains higher than $50 \%$ in the low voltage regime and decreases gradually as $\mathrm{V}>\mathrm{V}_{\text {th }}$. For the $5-\mu \mathrm{m}$ gel, $\mathrm{V}_{\text {th }}$ is about $7 \mathrm{~V}_{\text {rms. }}$. At $\mathrm{V}=30 \mathrm{~V}_{\mathrm{rms}}$, the measured contrast ratio for the green laser beam is as high as 230:1 when the distance of the detector is $40 \mathrm{~cm}$ from the cell.

To verify that the gel is indeed polarization independent, we rotated the cell by $90^{\circ}$ and repeated the voltage-dependant reflectance curves.

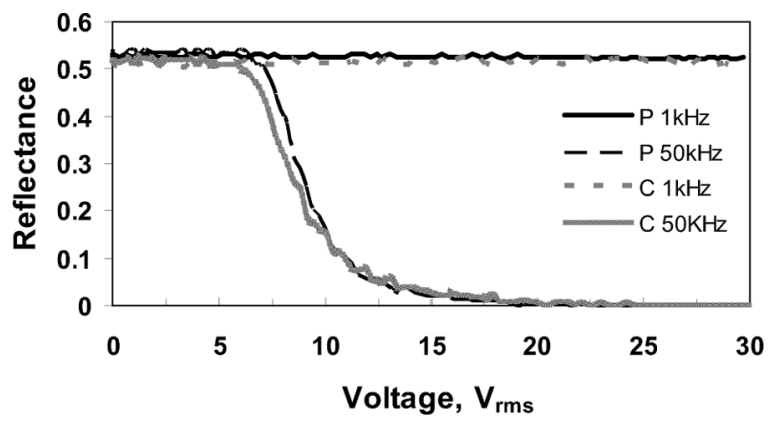

FIGURE 2 Voltage-dependent reflectance of dye-doped DFLC gel. P and C's polarizations of the incident light are orthogonal. The curves show that the device is polarization independent. 
Results are plotted in Figure 2, where $\mathrm{P}$ and $\mathrm{C}$ stand for two orthogonal polarization states. From Figure 2, the $\mathrm{P}$ and $\mathrm{C}$ curves almost overlap each other. That means our dye-doped DFLC gel is polarization independent. The contrast ratio $(\mathrm{CR})$ is defined as the ratio of reflectance at $\mathrm{V}=30 \mathrm{~V}_{\text {rms }}$ and 0 . The $\mathrm{CR}$ is $\sim 230: 1$ at $f=50 \mathrm{kHz}$ and the maximum reflectance is $\sim 50 \%$.

For a scattering device, the contrast ratio is dependent on the distance of the detector from the sample, as shown in Figure 3. In a handheld reflective display, a comfortable viewing distance is about 20-25 cm. To mimic this condition, we shortened the detecting distance from 40 to $20 \mathrm{~cm}$ and the measured contrast ratio is still 120:1. This result indicates that our gel has a very strong scattering property. The scattered light diverges quite fast.

In our sample, the dark state voltage is still too high $\left(\sim 30 \mathrm{~V}_{\mathrm{rms}}\right)$ to be driven by TFT. Using a higher $\Delta \varepsilon$ DFLC material, thinner cell gap, or lower monomer concentration would lower the operating voltage. The contrast ratio can be further improved by increasing the cell gap, monomer concentration, or dye concentration. However, increasing dye concentration would reduce display reflectance and lead to a slower response time, increasing polymer concentration would cause a higher operating voltage, and finally increasing cell gap would increase the operating voltage, reduce the voltage-off state reflectance, and lengthen the response time.

Figure 4 shows the reflectance spectra of dye-doped DFLC gel at 0 (black line) and $20 \mathrm{~V}_{\text {rms }}$ at $f=50 \mathrm{kHz}$ (gray line). The light source we used is standard white light source (Mikropack, DH-2000, UV-VISNIR). We used an iris and a lens to collimate the white light and expand the beam diameter to $\sim 4 \mathrm{~mm}$. A dielectric mirror was placed

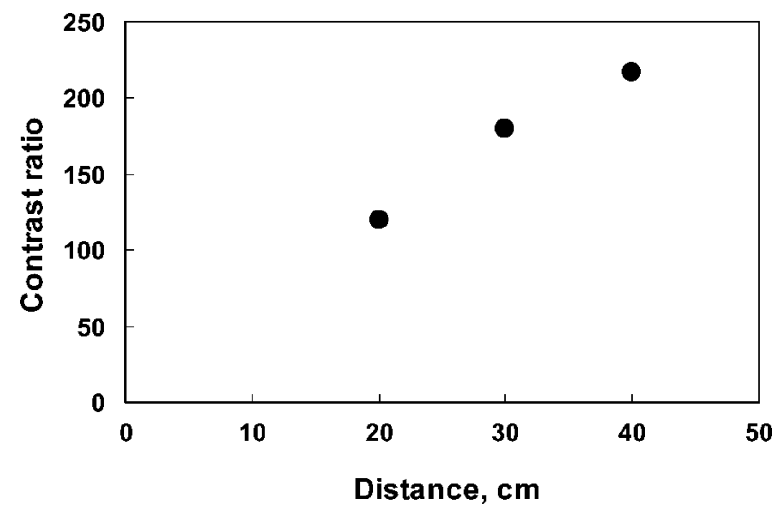

FIGURE 3 Contrast ratio as a function of detector's distance from sample. 


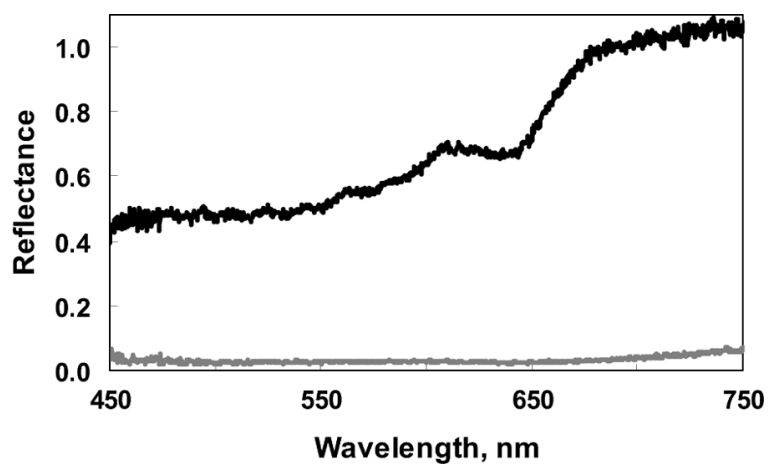

FIGURE 4 Reflectance spectrum of dye-doped DFLC gel at $0 \mathrm{~V}_{\text {rms }}$ (black line) and at $20 \mathrm{~V}_{\mathrm{rms}}(50 \mathrm{kHz})$ (gray line).

behind the LC cell for reflective mode measurement. The output beam was collected by a lens to a fiber-optics based universal serial bus (USB) spectrometer (resolution $=0.04 \mathrm{~nm}$; USB HR2000, Ocean Optics). The baseline we used for calibration was a pure LC cell with the same cell gap. In Figure 4 , at $\mathrm{V}=0$ the reflectance of the dyedoped DFLC gel is $\sim 50 \%$ between 450 and $550 \mathrm{~nm}$. Beyond $550 \mathrm{~nm}$, the reflectance increases because our dye-doped DFLC gel looks reddish, rather than black.

The dye-doped DFLC gel exhibits a good contrast ratio due to strong scattering and dye absorption. The reflectance $(\mathrm{R})$ can be expressed as

$$
R=e^{-\beta \cdot d \cdot N_{1}} e^{-\alpha \cdot c \cdot d \cdot N_{2}},
$$

where $\alpha$ is the average absorption coefficient, $\beta$ is the scattering coefficient, $c$ is the dye concentration $(\sim 0.05), d$ is the cell gap $(\sim 5 \mu \mathrm{m})$, and $N_{1}$ and $N_{2}$ are the scale numbers because of the multiple scattering and absorption. In Eq. (1), $\alpha$ is equal to $\alpha_{\|}$or $\alpha_{\perp}$ which stand for the absorption coefficients when the incident light polarization is parallel or perpendicular to the principal molecular axis of the dye molecules. At $\mathrm{V}=0$, the dye absorption $\left(\alpha_{\perp}\right)$ dominates and the gel's scattering is negligible. In a high voltage state at a high frequency, $\alpha$ can be expressed as:

$$
\alpha=\frac{\alpha_{\|}+\alpha_{\perp}}{2}
$$

because all the dye molecules are randomly oriented along the $x-y$ plane. In dye-doped PDLC, droplets are randomly dispersed in 3-dimensional space. So $\alpha$ is:

$$
\alpha=\frac{\alpha_{\|}+2 \alpha_{\perp}}{3}
$$



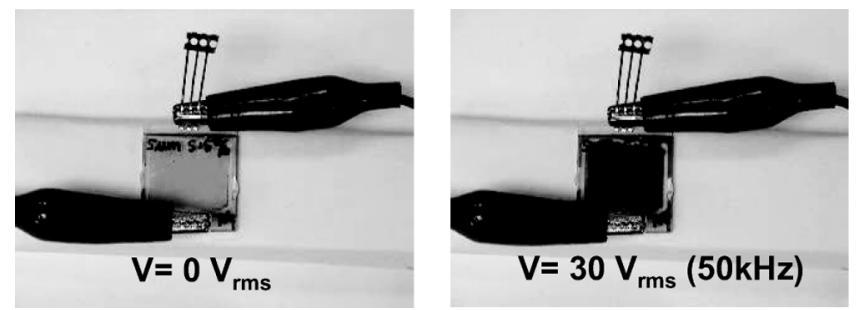

FIGURE 5 Single pixel of the 5 - $\mu \mathrm{m}$ dye-doped DFLC reflective display at $\mathrm{V}=0$ and $30 \mathrm{~V}_{\mathrm{rms}}(50 \mathrm{kHz})$.

By comparing Eq. (2) with Eq. (3), our dye-doped DFLC gel has a larger average absorption coefficient than the dye-doped PDLC. Due to the multi-domain structure and the random LC arrangement along the $\mathrm{x}-\mathrm{y}$ plane, the gel's scattering efficiency is maximized and independent of polarization. In addition, the dark state reflectance is minimized owing to the multiple light scattering in conjunction with dye absorption.

Response time is another important issue for guest-host displays. The dye molecules are usually bulky and have a high viscosity. Moreover, a guest-host display does not use any polarizer so that its governing response time equation is different from that with polarizer. As a result, a typical response time of a guest-host display is around $50 \mathrm{~ms}$. Detailed values depend on the dye concentration and cell gap. The response time of our dye-doped DFLC gel is fast. If we switch the applied voltage from 0 to $30 \mathrm{~V}_{\mathrm{rms}}$ at $50 \mathrm{kHz}$ frequency, the rise time is $1 \mathrm{~ms}$ and decay time is $10 \mathrm{~ms}$. If we fix the voltage at $30 \mathrm{~V}_{\mathrm{rms}}$ while switching the frequency between $1 \mathrm{kHz}$ and $50 \mathrm{kHz}$, the rise time is reduced to $\sim 0.55 \mathrm{~ms}$ and decay time to $\sim 5.78 \mathrm{~ms}$.

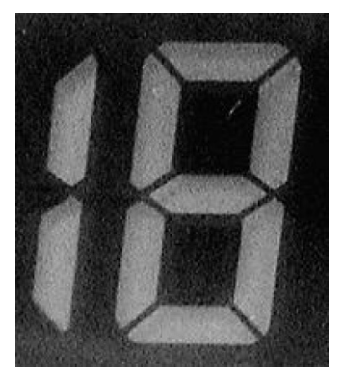

FIGURE 6 A demo of a segmented dye-doped DFLC reflective display. A diffusive reflector is laminated to the back of the bottom glass substrate. In the white segments, the ITO electrodes are etched away so that $\mathrm{V}=0$. Cell gap $=7 \mu \mathrm{m}$. 
Figure 5 shows a single pixel of the 5 - $\mu \mathrm{m}$ dye-doped DFLC reflective display at $0 \mathrm{~V}_{\mathrm{rms}}$ and $30 \mathrm{~V}_{\mathrm{rms}}(50 \mathrm{kHz})$. It shows good bright and dark states. To prove principle, we also fabricated a segmented reflective display using the dye-doped DFLC gel. Figure 6 shows a sample using a 7- $\mu \mathrm{m}$ dye-doped DFLC gel. To avoid specular reflection, we laminated a diffusive reflector on the backside of the bottom glass substrate in order to widen the viewing angle. The bright segments represent the areas without ITO electrodes. Since no voltage was applied, these segments appear white. The dark areas represent the ITO electrodes with $\mathrm{V}=30 \mathrm{~V}_{\text {rms }}$ at $f=50 \mathrm{kHz}$.

\section{CONCLUSION}

We have demonstrated a polarizer-free, high contrast, and fast response new reflective GH LCD using a dye-doped DFLC gel. The reflectance reaches $\sim 50 \%$ and the contrast ratio $>100: 1$. The response time is fast $(\sim 6 \mathrm{~ms})$. Since it does not require any polarizer, the viewing angle is wide. This new reflective GH LCD is attractive for handheld displays. To make color displays, pixilated color filters should be implemented. The major challenge of this dye-doped DFLC gel is to lower the driving voltage.

\section{REFERENCES}

[1] Bahadur, B. (1992). Liquid Crystals Applications and Uses, World Scientific: Singapore, Vol. 3, Ch. 11.

[2] Wu, S. T. \& Yang, D. K. (2001). Reflective Liquid Crystal Displays, Wiley: New York, Ch. 6.

[3] Cole, H. S. \& Kashnow, R. A. (1977). Appl. Phys. Lett., 30, 619.

[4] White, D. L. \& Taylor, G. N. (1974). J. Appl. Phys., 45, 4718.

[5] Uchida, T., Seki, H., Shishido, C., \& Wada, M. (1981). Proc. SID, 22, 41.

[6] Hasegawa, M., Takeda, K., Sakaguchi, Y., Egelhaaf, J., Lueder, E., Taira, Y., \& Lowe, A. C. (1999). SID Symposium Digest, 30, 962.

[7] Hasegawa, M., Hellermark, C., Nishikai, A., Taira, Y., \& Lowe, A. C. (2000). SID Symposium Digest, 31, 128.

[8] Fergason, J. L. (1985). SID Symposium Digest, 16, 68.

[9] Doane, J. W., Vaz, N. A., Wu, B. G., \& Zumer, S. (1986). Appl. Phys. Lett., 48, 269.

[10] Lin, Y. H., Ren, H., \& Wu, S. T. (2004). Appl. Phys. Lett., 84, 4083.

[11] Fan, Y. H., Ren, H., Liang, X., Lin, Y. H., \& Wu, S. T. (2004). Appl. Phys. Lett., 85, 2451.

[12] Lin, Y. H., Ren, H., Gauza, S., Wu, Y. H., Liang, X., \& Wu, S. T. (2005). J. Display Technology, 1, 230. 